\title{
Online parameter identification of synchronous machines using Kalman filter and recursive least squares
}

\author{
Erick F. Alves \\ Dept. of Electrical Power Engineering
NTNU
Trondheim, Norway
erick.f.alves@ntnu.no

\author{
Geir Mathisen \\ Dept. of Engineering Cybernetics \\ $N T N U$ \\ Trondheim, Norway \\ geir.mathisen@ntnu.no
}

\author{
Jonas K. Nøland \\ Dept. of Electrical Power Engineering \\ NTNU \\ Trondheim, Norway \\ jonas.k.noland@ntnu.no
}

\author{
Giancarlo Marafioti \\ Dept. of Mathematics and Cybernetics \\ SINTEF Digital \\ Trondheim, Norway \\ giancarlo.marafioti@sintef.no
}

\begin{abstract}
This paper investigates and implements a procedure for parameter identification of salient pole synchronous machines that is based on previous knowledge about the equipment and can be used for condition monitoring, online assessment of the electrical power grid, and adaptive control. It uses a Kalman filter to handle noise and correct deviations in measurements caused by uncertainty of instruments or effects not included in the model. Then it applies a recursive least squares algorithm to identify parameters from the synchronous machine model. Despite being affected by saturation effects, the proposed procedure estimates 8 out of 13 parameters from the machine model with minor deviations from data sheet values and is largely insensitive to noise and load conditions.
\end{abstract}

Index Terms-synchronous machines, parameter identification, condition monitoring

\section{INTRODUCTION}

Synchronous machines (SMs) are the bulk of power generation worldwide. In Norway, 95\% of the electricity production comes from hydro power [1], in which the use of salient pole, SMs is the norm. Therefore, the proper understanding of these devices is essential for planning, operation, and control of the power system [2]. Examples of tasks requiring adequate modeling and parametrization of SMs include load flow analysis, state estimation, stability assessment and tuning of grid controls and protection settings. These tasks are important for transmission system operators or generation companies to operate their resources optimally and reliably.

Traditionally, SM parameters are calculated by manufacturers in the design phase using detailed information of the machine [3], [4] or by recursive methods such as finiteelement analysis [5], [6]. Calculations are later validated during commissioning through acceptance or performance tests as described in IEEE and IEC Standards [7], [8]. These

The authors thank SINTEF Digital for supporting this research. methods for parameter identification are well-proven and have been used for decades to operate the power system reliably. However, they have two major shortcomings.

The first is considering that many parameter values in the system equations are constants and do not vary with time. However, several effects may impact the values of SM parameters over time. For example, the field current level determines the saturation of the magnetic core [9], and load conditions affects the magnetic equivalent air-gap length [10]. The historical reason for adopting such restrictions is simplifying equations and procedures for parameters calculation, due to the limited computational resources when the theory for SMs was developed.

The second shortcoming is requiring the machine to be in standstill or off-line for performing the majority of parameter estimation tests. Since this means loss of income for generation companies, tests are only executed during commissioning or planned stops. This limits greatly the amount of data and possible operational conditions that can be measured. In Norway, the transmission system operator (Statnett) requires the registration of generators' parameters for at least two weeks before commissioning, and an update with measured values after the machine starts commercial operation [11]. Yet, there is no requirement for periodical updates nor registration of distinct parameter values for different operational conditions.

Automated procedures for parameter identification of SMs have been overcoming some of these shortcomings in the last two decades [6], [12]-[14]. Those procedures were encouraged by the popularization of system identification techniques and their easy access in mathematical tools such as MATLAB ${ }^{\odot}$ [15]. Methods are varied, but approaches can be summarized in: 1) analysis of transient data, such as short-circuit or load rejections; 2) frequency response tests, with injection of perturbations in standstill, off-line or on line operation. Suc- 
cessful examples of such automated procedures are described in industry standards [2], [7].

This paper presents the following contributions: 1) the proposed automated procedure for parameter estimation can run with the machine online, and without taking it out of service, performing difficult and time-consuming tests or involving large perturbations; 2) the required input data are datasheet values and common measurements available in a power plant, i.e. there is no need to install additional transducers in the machine; 3 ) the algorithm is robust to noise and deviations in measurements caused by uncertainty of instruments or effects not included in the model.

\section{SYNCHRONOUS MACHINE MODEL}
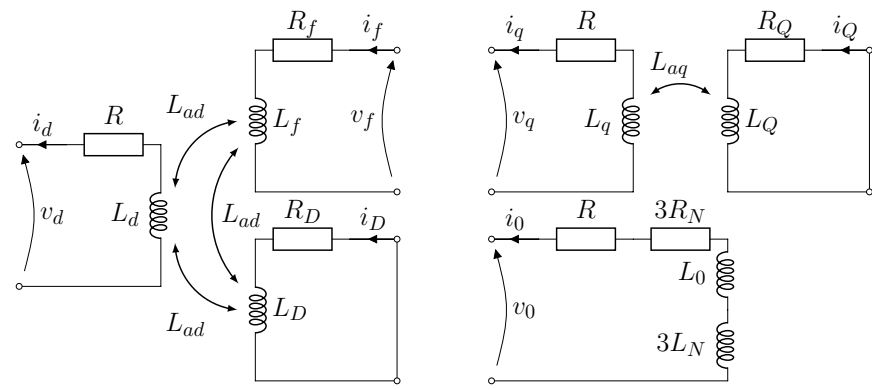

Fig. 1. Equivalent circuits of a synchronous machine in per unit and the dq0 reference frame

Fig. 1 presents the equivalent circuits of a SM in per unit and the $\mathrm{dq} 0$ reference frame. This model is thoroughly described in the literature, such as sections 11.1 and A.1 of [16], and will not be reviewed for the sake of brevity. Eq. (1) introduces the SM dynamic model and table I describes its variables.

$$
\mathbf{v}_{\text {dqOfDQ }}=-\mathbf{R}_{\text {sm }} \mathbf{i}_{\text {dqOfDQ }}-\mathbf{L}_{\mathbf{s m}} \frac{d}{d t} \mathbf{i}_{\mathbf{d q O f D Q}}
$$

where $\quad \mathbf{v}_{\mathbf{d q O f D Q}}=\left[\begin{array}{llllll}v_{d} & v_{q} & v_{0} & -v_{f} & 0 & 0\end{array}\right]^{T}$, $\mathbf{i}_{\mathbf{d q O f D Q}}=\left[\begin{array}{llllll}i_{d} & i_{q} & i_{0} & i_{f} & i_{D} & i_{Q}\end{array}\right]^{T}$, and

$\mathbf{R}_{\mathbf{s m}}=\left[\begin{array}{cccccc}R & \omega L_{q} & & & & \omega L_{a q} \\ -\omega L_{d} & R & & -\omega L_{a d} & -\omega L_{a d} & \\ & & R+3 R_{N} & R_{f} & & \\ & & & & R_{D} & \\ & & & & & R_{Q}\end{array}\right]$

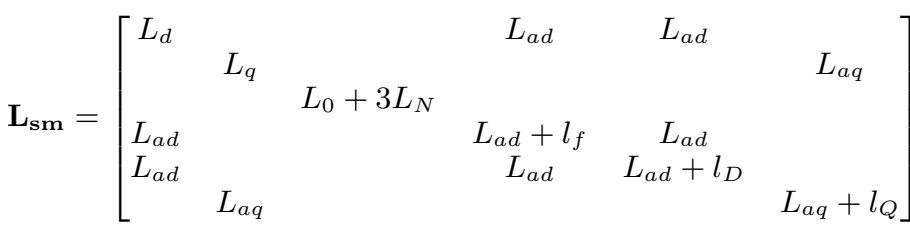

\section{A. State space representation}

By inspection of eq. (1), it would be natural to assume $\mathbf{u}=\mathbf{v}_{\text {dqOfDQ }}, \mathbf{y}=\mathbf{x}=\mathbf{i}_{\mathbf{d q O f D Q}}, \mathbf{A}=-\mathbf{L}_{\mathbf{s m}}{ }^{-1} \mathbf{R}_{\mathbf{s m}}$, $\mathbf{B}=-\mathbf{L}_{\mathbf{s m}}{ }^{-1}, \mathbf{C}=\mathbf{I}, \mathbf{D}=\mathbf{0}$. Nonetheless, it is more natural to assume $\mathbf{u}=\left[\begin{array}{llllll}i_{d} & i_{q} & i_{0} & v_{f} & 0 & 0\end{array}\right]^{T}$, since the armature currents are defined by loads and the field
TABLE I

DEFINITION OF SYMBOLS USED IN EQUATIONS AND FIGURES

\begin{tabular}{|c|c|c|c|}
\hline Var. & Definition & Var. & Definition \\
\hline$f_{n}$ & rated frequency & $l_{Q}$ & q-axis leakage inductance \\
\hline$i_{0}$ & zero sequence current & $L_{q}$ & $\mathrm{q}$-axis synchronous induct. \\
\hline$i_{d}$ & $\mathrm{~d}$-axis current & $p$ & pair of poles \\
\hline$i_{D}$ & $\begin{array}{l}\text { d-axis damper winding } \\
\text { current }\end{array}$ & $R$ & armature winding resist. \\
\hline$i_{f}$ & field current & $R_{D}$ & $\begin{array}{l}\text { d-axis damper } \\
\text { winding resist. }\end{array}$ \\
\hline$I_{f n}$ & rated field current & $R_{f}$ & field winding resist. \\
\hline$i_{q}$ & $\mathrm{q}$-axis current & $R_{N}$ & neutral grounding resist. \\
\hline$i_{Q}$ & $\begin{array}{l}\text { q-axis damper winding } \\
\text { current }\end{array}$ & $R_{Q}$ & $\begin{array}{l}\text { q-axis damper winding } \\
\text { resist. }\end{array}$ \\
\hline$L_{0}$ & zero sequence induct. & $S_{n}$ & $\begin{array}{l}\text { rated armature } \\
\text { apparent power }\end{array}$ \\
\hline$L_{a d}$ & $\begin{array}{l}\mathrm{d} \text {-axis armature } \\
\text { magnetizing induct. }\end{array}$ & $U_{n}$ & $\begin{array}{l}\text { rated armature } \\
\text { line-to-line voltage }\end{array}$ \\
\hline$L_{a q}$ & $\begin{array}{l}\text { q-axis armature } \\
\text { magnetizing induct. }\end{array}$ & $v_{0}$ & zero sequence voltage \\
\hline$l_{D}$ & d-axis leakage induct. & $v_{d}$ & $\mathrm{~d}$-axis voltage \\
\hline$L_{d}$ & $\begin{array}{l}\text { d-axis synchronous } \\
\text { induct. }\end{array}$ & $v_{f}$ & field voltage \\
\hline$l_{f}$ & field leakage induct. & $v_{q}$ & q-axis voltage \\
\hline$l_{l}$ & armature leakage induct. & $Z_{b}$ & base impedance $=\frac{U_{n}^{2}}{S_{n}}$ \\
\hline$L_{N}$ & neutral grounding induct. & $\omega$ & rotor speed \\
\hline
\end{tabular}

voltage is delivered by the excitation system. Thus, $\mathbf{y}=$ $\left[\begin{array}{llllll}v_{d} & v_{q} & v_{0} & i_{f} & i_{D} & i_{Q}\end{array}\right]^{T}$ and matrices $\mathbf{A}, \mathbf{B}, \mathbf{C}, \mathbf{D}$ must be redefined for a proper state-space representation.

This can be achieved without major changes to the structure of eq. (1) by extending the model presented in fig. 1 [17]. Let suppose a balanced, star-connected load with resistance $R_{d l}=10^{4} Z_{b}$ is inserted at the machine terminals, as shown in fig. 2. This dummy load with very high resistance can be understood as a measurement device. With that, the armature voltages in the $(\mathrm{d}, \mathrm{q}, 0)$ reference frame can be expressed as $v_{d}=R_{d l}\left(i_{d}-i_{d t}\right), v_{q}=R_{d l}\left(i_{q}-i_{q t}\right), v_{0}=R_{d l}\left(i_{0}-i_{0 t}\right)$.
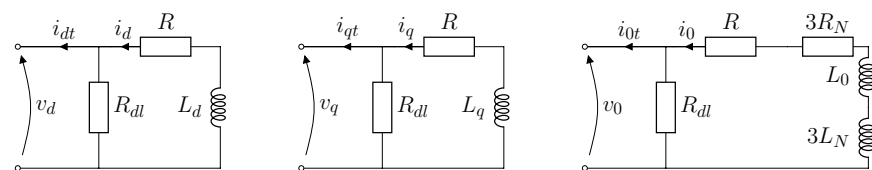

Fig. 2. Synchronous machine with a resistance $R_{d l}$ connected at its terminals

Notice that $R_{d l}$ is considerably larger than the real load of the machine, therefore the difference between the terminal currents $i_{d t}, i_{q t}, i_{0 t}$ and armature currents $i_{d}, i_{q}, i_{0}$ is negligible. Re-arranging eq. (1) with these considerations, one obtains:

$$
\mathbf{v}_{\text {dl }}=-\mathbf{R}_{\text {smdl }} \mathbf{i}_{\text {dqOfDQ }}-\mathbf{L}_{\text {sm }} \frac{d}{d t} \mathbf{i}_{\text {dqOfDQ }}
$$

where $\mathbf{v}_{\mathbf{d l}}=\left[\begin{array}{llllll}R_{d l} i_{d t} & R_{d l} i_{q t} & R_{d l} i_{0 t} & v_{f} & 0 & 0\end{array}\right]^{T}$ and $\mathbf{R}_{\mathrm{smdl}}=$

$\left[\begin{array}{cccccc}R+R_{d l} & \omega L_{q} & & & \omega L_{a q} \\ -\omega L_{d} & R+R_{d l} & & -\omega L_{a d} & -\omega L_{a d} & \\ & & R+3 R_{N}+R_{d l} & R_{f} & & \\ & & & & R_{D} & \\ & & & & & R_{Q}\end{array}\right]$ 
Hence, the following state space is defined:

$$
\begin{aligned}
& \mathbf{u}=\mathbf{v}_{\mathbf{d l}} \quad \mathbf{x}=\mathbf{i}_{\mathbf{d q O f D Q}} \\
& \mathbf{y}=\left[\begin{array}{llllll}
v_{d} & v_{q} & v_{0} & i_{f} & i_{D} & i_{Q}
\end{array}\right]^{T} \\
& \mathbf{A}=-\mathbf{L}_{\mathbf{s m}}{ }^{-1} \mathbf{R}_{\text {smdl }} \quad \mathbf{B}=\mathbf{L}_{\mathbf{s m}}{ }^{-1} \\
& \mathbf{C}=\operatorname{diag}\left[\begin{array}{llllll}
R_{d l} & R_{d l} & R_{d l} & 1 & 1 & 1
\end{array}\right] \\
& \mathbf{D}=\operatorname{diag}\left[\begin{array}{llllll}
-1 & -1 & -1 & 0 & 0 & 0
\end{array}\right]
\end{aligned}
$$

It is quite uncommon to measure the damper winding currents of a SM. For this reason, a transfer function for the observer for damper windings (ODW) currents is derived by applying the Laplace transform to rows 5 and 6 of eq. (2), and manipulating the expressions further to obtain:

$$
\begin{aligned}
& i_{D}=-\frac{s L_{a d}}{s\left(L_{a d}+l_{D}\right)+R_{D}}\left(i_{d}+i_{f}\right) \\
& i_{Q}=-\frac{s L_{a q}}{s\left(L_{a q}+l_{Q}\right)+R_{Q}} i_{q}
\end{aligned}
$$

\section{OPTIMAL OBSERVER WITH KALMAN FILTER}

In the proposed procedure, a Kalman filter (KF) corrects and filters measurements using prior knowledge about the SM model and its parameters. In addition to eq. (3), three additional matrices must be specified to define a KF [18]:

- Q, a SxS matrix ( $\mathrm{S}=$ number of states) in which the diagonal elements represents the noise covariance of the states, also called process noise covariance matrix;

- $\mathbf{R}$, a YxY matrix (Y=number of outputs) in which the diagonal elements represents the noise covariance of the outputs, also called measurement noise covariance matrix;

- N, a SxY matrix in which the elements represents the noise cross-covariance between states and outputs, also called process and measurement noise cross-covariance matrix.

It is assumed $\mathbf{N}=\mathbf{0}$, i.e. the noise is white and there is no cross-correlation between the noise of states and outputs. The other matrices are empirically defined as $\mathbf{Q}=\operatorname{diag}\left[\begin{array}{llllll}0.05 & 0.05 & 0.05 & 0.05 & 0.03 & 0.03\end{array}\right]$ and $\mathbf{R}=$ diag $\left[\begin{array}{llllll}0.05 & 0.05 & 0.05 & 0.05 & 0.05 & 0.05\end{array}\right]$. Notice that the choice of diagonal $\mathbf{Q}$ and $\mathbf{R}$ matrices represents a naive assumption that state and output changes are uncorrelated.

These values produce robust results in several load conditions with and without noise, as seen in table III. However, fine tuning might be required for better performance, according to the level of noise, measurement accuracy and precision in the field. In practice, these values are also affected by the variance of $\mathbf{A}, \mathbf{B}, \mathbf{C}, \mathbf{D}$ elements.

\section{A. Model validation}

A simulation in MATLAB/Simulink is implemented to validate the $\mathrm{KF}$ and the ODW. It contains a load connected to a SM model in pu from the Simscape Power Systems (SPS) library [19], which is used as benchmark. Parameters of a real synchronous machine are taken from examples 3.1, 3.2 and 8.1 of [20] and listed in table II. Saturation effects are included in the SPS SM model.

TABLE II

PARAMETERS OF THE BENCHMARK SYNCHRONOUS MACHINE

\begin{tabular}{ll|ll}
\hline Parameter & Value & Parameter & Value \\
\hline$S_{n}$ & $555 \mathrm{MVA}$ & $L_{a d}$ & $1.66 \mathrm{pu}$ \\
$U_{n}$ & $24 \mathrm{kV}$ & $L_{a q}$ & $1.61 \mathrm{pu}$ \\
$f_{n}$ & $60 \mathrm{~Hz}$ & $l_{D}$ & $0.1713 \mathrm{pu}$ \\
$I_{f n}$ & $1300 \mathrm{~A}$ & $l_{Q}$ & $0.7252 \mathrm{pu}$ \\
$p$ & $1 \mathrm{pair}$ & $R_{f}$ & $0.0006 \mathrm{pu}$ \\
$R$ & $0.003 \mathrm{pu}$ & $l_{f}$ & $0.165 \mathrm{pu}$ \\
$l_{l}$ & $0.15 \mathrm{pu}$ & & \\
\hline
\end{tabular}

The rotor speed is assumed constant, i.e. the prime mover and its turbine governor are not modeled because the mechanical dynamics are much slower than the electrical and have little influence in the results. The field voltage is provided by an DC1C type excitation system as described in [21]. The choice of parameters for the automatic voltage regulator (AVR) gives a fast and stable response, without overshoot in the terminal voltage.

The outputs of the SM, i.e. armature and field measurements $v_{a}, v_{b}, v_{c}, v_{f}, i_{a}, i_{b}, i_{c}, i_{f}$ together with the rotor mechanical angle $\gamma$, are fed into a measurement block that: 1) adds band-limited white noise and re-samples measurements into a lower sample frequency $(400 \mathrm{~Hz})$ in order to make them more realistic; 2) applies the $d q 0$-transformation and converts the values to per-unit. Finally, the output of the measurement block is fed into the proposed ODW and KF.

The simulation runs with the following load conditions, where $\mathrm{P}$ represent the active power, $\mathrm{Q}$ the reactive power and the per-unit base is given in table II:

- $\underline{\text { Case 1: }} \mathrm{P}=0 \mathrm{pu}, \mathrm{Q}=0 \mathrm{pu}$ (no load);

- Case 2: $\mathrm{P}=0.5 \mathrm{pu}, \mathrm{Q}=0.5 \mathrm{pu}$;

- Case 3: $\mathrm{P}=0.5 \mathrm{pu}, \mathrm{Q}=-0.5 \mathrm{pu}$;

- Case 4: $\mathrm{P}=0.9 \mathrm{pu}, \mathrm{Q}=0.4359 \mathrm{pu}$ (rated load).

In addition, the following noise power density $\left(N_{p}\right)$ scenarios are used for each simulation case: no noise $N_{p}=0$, standard noise $N_{p}=10^{-10} \mathrm{~W} / \mathrm{Hz}$, high noise $N_{p}=10^{-9} \mathrm{~W} / \mathrm{Hz}$. Those noise scenarios correspond respectively to signal-tonoise ratio (SNR) equal to $\infty, 50 \mathrm{~dB}$ and $40 \mathrm{~dB}$ when the measured signal is $1 \mathrm{pu}$.

In all cases and noise scenarios, the simulation starts at rated armature voltage. In order to observe transient behavior, a step of $+5 \%$ is applied to the reference of the AVR at time $t=17$ seconds. The initial states of the SM are calculated using the Machine Initialization tool from SPS in order to avoid loss of synchronism [19]. However, initial states of the ODW and the KF are zero, so it is necessary some seconds of simulation to achieve steady state. This is done to demonstrate the KF robustness to wrong initial conditions and large transients.

Table III benchmarks the proposed KF against the SPS SM by presenting the goodness of fit between the two models. For that, it uses the normalized mean square error (NMSE) as cost 
function which is defined as:

$$
N M S E=1-\frac{\left\|x_{r e f}-x\right\|^{2}}{\left\|x_{r e f}-\bar{x}_{r e f}\right\|^{2}}
$$

where $\|$ indicates the Euclidean or $L^{2}$ norm of a vector. NMSE costs vary between $-\infty$ (bad fit) to 1 (perfect fit).

Note that the NMSE of $v_{d}$ and $i_{Q}$ are very low in case 1 (no load) because their values tend to zero and, since the noise power is constant, the SNR is extremely low. This makes NMSE measurement not relevant for these cases, so they are excluded from the standard deviation (std dev) calculation.

TABLE III

NMSE VALUES FOR ALL SIMULATION CASES AND NOISE SCENARIOS WITH SATURATION

\begin{tabular}{lrrrrrr}
\hline Variable & Case 1 & Case 2 & Case 3 & Case 4 & Mean & Std dev \\
\hline \multicolumn{7}{c}{ No noise scenario } \\
\hline$v_{d}$ KF & 1.000 & 1.000 & 1.000 & 1.000 & 1.000 & $9.93 \mathrm{e}-10$ \\
$v_{q} \mathrm{KF}$ & 1.000 & 1.000 & 1.000 & 1.000 & 1.000 & $2.39 \mathrm{e}-09$ \\
$i_{f} \mathrm{KF}$ & 1.000 & 1.000 & 1.000 & 1.000 & 1.000 & $8.56 \mathrm{e}-06$ \\
$i_{D} \mathrm{ODW}$ & 0.609 & 0.637 & 0.997 & 0.975 & 0.804 & $2.10 \mathrm{e}-01$ \\
$i_{D} \mathrm{KF}$ & 0.612 & 0.620 & 0.995 & 0.964 & 0.798 & $2.11 \mathrm{e}-01$ \\
$i_{Q} \mathrm{ODW}$ & 1.000 & 1.000 & 1.000 & 1.000 & 1.000 & $6.43 \mathrm{e}-08$ \\
$i_{Q} \mathrm{KF}$ & -437 & 0.995 & 1.000 & 0.999 & 0.998 & $2.66 \mathrm{e}-03$ \\
\hline \multicolumn{7}{c}{ Standard noise scenario } \\
\hline$v_{d} \mathrm{KF}$ & -489 & 0.954 & 0.996 & 0.984 & 0.978 & $2.18 \mathrm{e}-02$ \\
$v_{q} \mathrm{KF}$ & 0.996 & 0.994 & 0.988 & 0.988 & 0.991 & $4.13 \mathrm{e}-03$ \\
$i_{f} \mathrm{KF}$ & 0.999 & 1.000 & 0.995 & 0.999 & 0.998 & $2.34 \mathrm{e}-03$ \\
$i_{D} \mathrm{ODW}$ & 0.448 & 0.431 & 0.907 & 0.753 & 0.635 & $2.34 \mathrm{e}-01$ \\
$i_{D} \mathrm{KF}$ & 0.559 & 0.551 & 0.965 & 0.889 & 0.741 & $2.17 \mathrm{e}-01$ \\
$i_{Q} \mathrm{ODW}$ & -5263 & 0.859 & 0.991 & 0.932 & 0.927 & $6.63 \mathrm{e}-02$ \\
$i_{Q} \mathrm{KF}$ & -2305 & 0.945 & 0.997 & 0.975 & 0.972 & $2.59 \mathrm{e}-02$ \\
\hline \multicolumn{7}{c}{ High noise scenario } \\
\hline$v_{d} \mathrm{KF}$ & -4890 & 0.540 & 0.963 & 0.844 & 0.782 & $2.18 \mathrm{e}-01$ \\
$v_{q} \mathrm{KF}$ & 0.961 & 0.937 & 0.884 & 0.876 & 0.915 & $4.13 \mathrm{e}-02$ \\
$i_{f} \mathrm{KF}$ & 0.988 & 0.995 & 0.946 & 0.994 & 0.981 & $2.33 \mathrm{e}-02$ \\
$i_{D} \mathrm{ODW}$ & -1.005 & -1.437 & 0.096 & -1.253 & -0.900 & $6.87 \mathrm{e}-01$ \\
$i_{D} \mathrm{KF}$ & 0.066 & -0.080 & 0.692 & 0.211 & 0.222 & $3.35 \mathrm{e}-01$ \\
$i_{Q} \mathrm{ODW}$ & -52633 & -0.414 & 0.909 & 0.321 & 0.272 & $6.63 \mathrm{e}-01$ \\
$i_{Q} \mathrm{KF}$ & -19133 & 0.496 & 0.968 & 0.759 & 0.741 & $2.37 \mathrm{e}-01$ \\
\hline
\end{tabular}

\section{Algorithm FOR PARAMETER ESTIMATION}

Eq. (1) shows that, in matricial form, a synchronous machine can be reduced to an impedance with a resistive part $\mathbf{R}_{\mathbf{s m}}$ and an inductive part $\mathbf{L}_{\mathbf{s m}}$. Given this model structure and the set of process signal $\mathbf{v}_{\mathbf{d q O f D Q}}, \mathbf{i}_{\mathbf{d q O f D Q}}$, the goal is to estimate the elements of matrices Rsm, Lsm. For that, an approximation or error criterion is required.

The literature has some accounts of error criteria for parameter identification of synchronous machines, such as KF [22], recursive least squares (RLS) [12], [23], Prony method [24], among others.

In this paper, the error criterion applied is the RLS. The main reasons for this choice are: 1) RLS is readily available in the System Identification Toolbox of Simulink; 2) near real-time execution is possible with RLS due to its recursive nature and low computational effort when compared to other methods. This is essential when considering direct implementation in existing intelligent electronic devices (IEDs) or phasor measurement units (PMUs); 3) benchmarks exist in the literature for comparison of results.

Considering simultaneous estimation of the 13 parameters of the synchronous machine with RLS estimation generates poor results [12], simplifications are required. Thus, steadystate is assumed, i.e. $\frac{d}{d t} \mathbf{i}_{\text {dq0fDQ }}=\mathbf{0}$. Therefore, parameters from matrix $\mathbf{R}_{\mathrm{sm}}$ can be estimated using RLS, but not $\mathbf{L}_{\mathbf{s m}}$. However, notice that 4 out of 8 parameters from $\mathbf{L}_{\mathrm{sm}}$ are also present in $\mathbf{R}_{\mathrm{sm}}$.

Another practical simplification is to remove the armature resistance $R$ from rows 1 and 2 of matrix $\mathbf{R}_{\text {smdl }}$. The arguments for this are: 1) $R$ is not used for the calculation of any standard parameter of the SM (refer to table IV); 2) $R$ in pu is usually two to three orders of magnitude smaller than other parameters in these rows $\left(\omega L_{d}, \omega L_{a d}, \omega L_{q}, \omega L_{a q}\right)$, what makes a reliable estimation challenging [25].

Considering all assumptions above, eq. (1) can be rearranged into:

$$
\mathbf{v}_{\mathbf{R L S}}=-\mathbf{R}_{\mathbf{R L S}} \mathbf{i}_{\text {dqOfDQ }}
$$

where $\mathbf{v}_{\mathbf{R L S}}=\left[\begin{array}{llllll}v_{d}-R i_{d} & v_{q}-R i_{q} & v_{0} & -v_{f} & 0 & 0\end{array}\right]^{T}$ and

$$
\mathbf{R}_{\mathbf{R L S}}=\left[\begin{array}{cccccc} 
& \omega L_{q} & & & & \omega L_{a q} \\
-\omega L_{d} & & & -\omega L_{a d} & -\omega L_{a d} & \\
& & R+3 R_{N} & R_{f} & & \\
& & & R_{D} & \\
& & & & R_{Q}
\end{array}\right]
$$

Notice that in $\mathbf{V}_{\mathbf{R L S}}$, the armature voltages $v_{d}, v_{q}$ are compensated with the voltage drops in the armature resistance $R i_{d}, R i_{q}$. Also $R+3 R_{N}$ is estimated in the third row. In summary, eq. (1) is only re-arranged to avoid the estimation of $R$ individually.

The leakage reactances $l_{f}, l_{D}, l_{Q}$ are not estimated by the RLS algorithm. Hence, they are assumed constants for calculation of standard parameters. This is reasonable considering they represent a flux path through air and are less affected by saturation or temperature changes.

Finally, the steady-state condition is detected in run-time by monitoring that damper windings currents are below a certain level, as those flow only in transient conditions. Fine tuning in the field of this threshold might be required for better performance, according to the noise level, measurement accuracy and precision of each application.

For validation of the parameter estimator, the simulation file runs at exactly the same conditions as described in sec. III-A. The output of the KF is fed into six RLS estimators, i.e. one for each line of $\mathbf{R}_{\mathbf{R L S}}$, as seen in fig. 3. The estimators use a memory time of 3 seconds and sampling frequency of 400 $\mathrm{Hz}$. Results are evaluated measuring the error in percentage from values in table II.

Table V presents a summary of these evaluations. Results are shown before and after the AVR step in order to 


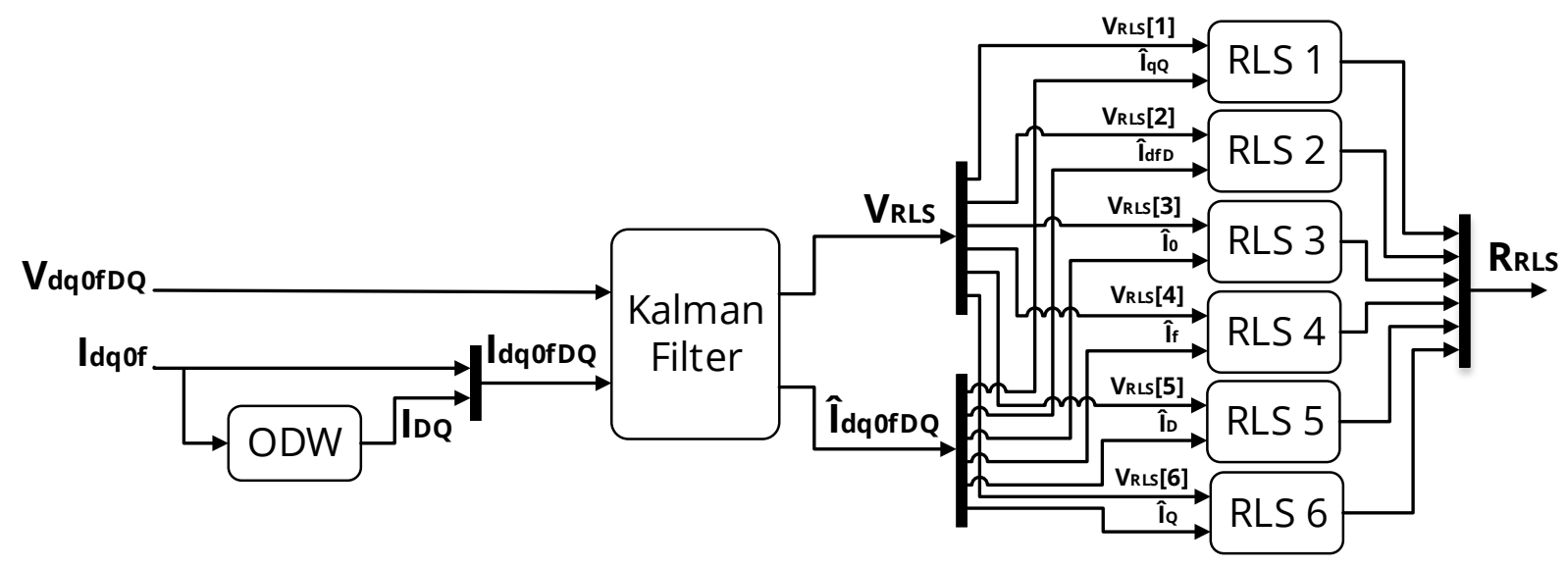

Fig. 3. Overview of the proposed parameter identification procedure

TABLE IV

STANDARD PARAMETERS OF A SALIENT-POLE SYNCHRONOUS MACHINE [20]

\begin{tabular}{ll}
\hline Parameter & Definition \\
\hline$X_{d}$ & $\omega\left(L_{a d}+l_{l}\right)$ \\
$T_{d 0}^{\prime}$ & $\frac{L_{a d}+l_{f}}{R_{f}}+\frac{L_{a d}+l_{D}}{R_{D}}$ \\
$T_{d}^{\prime}$ & $\frac{1}{R_{f}}\left(l_{f}+\frac{L_{a d} l_{l}}{L_{a d}+l_{l}}\right)+\frac{1}{R_{D}}\left(l_{D}+\frac{L_{a d} l_{l}}{L_{a d}+l_{l}}\right)$ \\
$T_{d 0}^{\prime \prime}$ & $\frac{1}{T_{d 0}^{\prime} R_{f} R_{D}}\left(l_{D}+\frac{L_{a d} l_{f}}{L_{a d}+l_{f}}\right)\left(L_{a d}+l_{f}\right)$ \\
$T_{d}^{\prime \prime}$ & $\frac{1}{T_{d}^{\prime} R_{f} R_{D}}\left(l_{D}+\frac{L_{a d} l_{l} l_{f}}{L_{a d} l_{l}+L_{a d} l_{f}+l_{l} l_{f}}\right)\left(l_{f}+\frac{L_{a d} l_{l}}{L_{a d}+l_{l}}\right)$ \\
$X_{d}^{\prime}$ & $X_{d} \frac{T_{d}^{\prime}}{T_{d 0}^{\prime}}$ \\
$X_{d}^{\prime \prime}$ & $X_{d}^{\prime} \frac{T_{d}^{\prime \prime}}{T_{d 0}^{\prime \prime}}$ \\
$X_{q}$ & $\omega\left(L_{a q}+l_{l}\right)$ \\
$T_{q 0}^{\prime \prime}$ & $\frac{\left(L_{a q}+l_{Q}\right)}{R_{Q}}$ \\
$X_{q}^{\prime \prime}$ & $\omega\left(l_{l}+\frac{L_{a q} l_{Q}}{L_{a q}+l_{Q}}\right)$ \\
\hline
\end{tabular}

demonstrate the effectiveness of the steady-state detection and robustness of the procedure to transient effects.

\section{DiscusSiON}

Table III points to a mean correlation close to unity between $\mathrm{KF}$ and SPS in the no noise scenario for all variables except $i_{D}$. Also in the standard noise scenario, correlation between $\mathrm{KF}$ and SPS is relatively close to unity and with small std dev, except for $i_{D}$. This shows the two models are nearly equivalent.

Note that saturation changes the value of $L_{a d}$, which is the main component of the zero and pole of $i_{D}$ transfer function in the ODW, as shown in eq. (4). This variation of $L_{a d}$ makes the state transition function non linear, and improper for a KF to handle. The main result is that the KF does not compensate saturation for $i_{D}$. On the other hand, the KF effectively compensates saturation for $v_{q}, i_{f}$ in the no and standard noise scenarios. As expected, performance is degraded in the high noise scenario due to a lower SNR.

Therefore, saturation effects must be considered and included in future work. An alternative for that would be using an extended or unscented KF, which can handle non linear state transition functions, and compensate the value of $L_{a d}$ dynamically [26]. Alternatively, a more advanced non linear model of the machine including saturation in its derivation can be used [27], [28].

Moreover, the KF improves $i_{D}, i_{Q}$ measurements considerably when the machine load increases, due to a better SNR. This is very noticeable in the standard and high noise scenario. Not least, notice in table III that the low std dev between all cases indicates the correlation is not sensitive to the load conditions.

As seen in table $\mathrm{V}$, the proposed procedure summarized in fig. 3 estimates parameters of the SPS SM machine with relative small percentage deviations from the datasheet values, and are in line with those reported in the literature [12]. The low std dev between all cases indicates the estimation is not sensitive to the load connected to the machine. In addition, noise power has small influence in the quality of the parameter estimation. This seems to be an advantage of the KF over other filtering techniques reported in the literature [12], [23]. However, this finding must be corroborated by experimental results in a real SM to prove its value.

The proposed strategy to disable and reset the RLS algorithm during transients is successful, as results are similar before and after the AVR step. No instability is observed in the estimated parameters for all cases and noise scenarios, also for long time simulations of 300 seconds in all noise scenarios (not included in the results for sake of brevity).

Nonetheless, note that the errors of $\omega L_{d}, \omega L_{a d}$ are considerable due to the saturation effect. Surprisingly, there is no direct correlation between the amplitude of this deviation 
TABLE V

PERCENTAGE ERRORS IN THE LAST 5 SECONDS OF ESTIMATION USING DATA SHEET VALUES AS BASELINE - BEFORE AND AFTER THE STEP

\begin{tabular}{|c|c|c|c|c|c|c|c|c|c|c|c|c|c|c|c|c|}
\hline \multirow[t]{3}{*}{ Param. } & \multicolumn{4}{|c|}{ Case 1} & \multicolumn{4}{|c|}{ Case 2} & \multicolumn{4}{|c|}{ Case 3} & \multicolumn{4}{|c|}{ Case 4} \\
\hline & \multicolumn{2}{|c|}{ mean } & \multicolumn{2}{|c|}{ std dev } & \multicolumn{2}{|c|}{ mean } & \multicolumn{2}{|c|}{ std dev } & \multicolumn{2}{|c|}{ mean } & \multicolumn{2}{|c|}{ std dev } & \multicolumn{2}{|c|}{ mean } & \multicolumn{2}{|c|}{ std dev } \\
\hline & before & after & before & after & before & after & before & after & before & after & before & after & before & after & before & after \\
\hline \multicolumn{17}{|c|}{ No noise scenario } \\
\hline$\omega L_{d}$ & 0.00 & 0.00 & 0.00 & 0.00 & -1.63 & -0.90 & 0.00 & 0.00 & -4.04 & -4.03 & 0.00 & 0.01 & -3.26 & -2.96 & 0.00 & 0.00 \\
\hline$\omega L_{q}$ & 0.26 & -0.01 & 0.00 & 0.00 & -0.87 & -0.97 & 0.03 & 0.01 & -0.22 & -0.26 & 0.01 & 0.00 & -0.79 & -0.85 & 0.02 & 0.00 \\
\hline$R+3 R_{N}$ & 0.00 & 0.00 & 0.00 & 0.00 & 0.00 & 0.00 & 0.00 & 0.00 & 0.00 & 0.00 & 0.00 & 0.00 & 0.00 & 0.00 & 0.00 & 0.00 \\
\hline$\omega L_{a d}$ & -0.08 & -3.51 & 0.01 & 0.01 & 3.26 & 1.84 & 0.00 & 0.00 & 5.17 & 5.17 & 0.01 & 0.01 & 5.20 & 4.76 & 0.00 & 0.00 \\
\hline$R_{f}$ & 0.52 & 0.02 & 0.10 & 0.00 & 0.00 & -0.04 & 0.00 & 0.01 & 0.00 & -0.33 & 0.00 & 0.09 & 0.00 & -0.07 & 0.00 & 0.02 \\
\hline$R_{D}$ & -0.01 & 0.00 & 0.00 & 0.00 & 0.00 & 0.00 & 0.00 & 0.00 & 0.00 & 0.00 & 0.00 & 0.00 & 0.00 & 0.00 & 0.00 & 0.00 \\
\hline$\omega L_{a q}$ & 0.02 & 0.00 & 0.00 & 0.00 & -0.02 & 0.00 & 0.00 & 0.00 & -0.02 & 0.00 & 0.00 & 0.00 & -0.02 & 0.00 & 0.00 & 0.00 \\
\hline$R_{Q}$ & 0.00 & 0.00 & 0.00 & 0.00 & -0.02 & -0.02 & 0.00 & 0.00 & -0.02 & 0.00 & 0.00 & 0.00 & -0.02 & -0.01 & 0.00 & 0.00 \\
\hline$T_{d 0}^{\prime}$ & -0.54 & -1.65 & 0.10 & 0.00 & 1.51 & 0.89 & 0.00 & 0.01 & 2.40 & 2.73 & 0.00 & 0.08 & 2.41 & 2.28 & 0.00 & 0.02 \\
\hline$T_{d}^{\prime, ~}$ & -0.34 & -0.05 & 0.10 & 0.00 & -0.61 & -0.66 & 0.02 & 0.02 & -0.12 & 0.17 & 0.01 & 0.09 & -0.53 & -0.53 & 0.01 & 0.02 \\
\hline$T_{d 0}^{\prime \prime}$ & -0.03 & -1.60 & 0.00 & 0.00 & 1.44 & 0.82 & 0.00 & 0.00 & 2.27 & 2.28 & 0.00 & 0.00 & 2.28 & 2.09 & 0.00 & 0.00 \\
\hline$T_{d}^{\prime \prime}$ & 1.01 & -0.16 & 0.02 & 0.01 & -3.63 & -4.19 & 0.13 & 0.04 & -0.69 & -0.96 & 0.04 & 0.00 & -3.20 & -3.57 & 0.07 & 0.02 \\
\hline$\stackrel{a}{X_{d}^{\prime}}$ & 0.35 & -1.65 & 0.01 & 0.01 & 0.03 & -0.81 & 0.05 & 0.02 & 1.98 & 1.88 & 0.01 & 0.00 & 1.03 & 0.70 & 0.03 & 0.01 \\
\hline$X_{d}^{\prime \prime}$ & 1.41 & -0.22 & 0.02 & 0.02 & -4.97 & -5.74 & 0.18 & 0.06 & -0.97 & -1.34 & 0.06 & 0.00 & -4.39 & -4.89 & 0.10 & 0.02 \\
\hline$T_{q 0}^{\prime \prime}$ & 0.02 & 0.00 & 0.00 & 0.00 & 0.01 & 0.02 & 0.00 & 0.00 & 0.01 & 0.00 & 0.00 & 0.00 & 0.01 & 0.01 & 0.00 & 0.00 \\
\hline$X_{q}^{q 0}$ & 0.64 & -0.03 & 0.01 & 0.01 & -2.32 & -2.63 & 0.08 & 0.03 & -0.54 & -0.70 & 0.03 & 0.00 & -2.09 & -2.30 & 0.05 & 0.01 \\
\hline & & & & & & & tandard $r$ & ise sce & ario & & & & & & & \\
\hline$\omega L_{d}$ & -0.02 & -0.01 & 0.00 & 0.00 & -1.64 & -0.91 & 0.00 & 0.00 & -4.04 & -4.04 & 0.00 & 0.01 & -3.26 & -2.97 & 0.00 & 0.00 \\
\hline$\omega L_{q}$ & 0.22 & -0.02 & 0.01 & 0.01 & -0.88 & -0.97 & 0.03 & 0.01 & -0.22 & -0.26 & 0.01 & 0.00 & -0.79 & -0.85 & 0.02 & 0.00 \\
\hline$R+3 R_{N}$ & -0.06 & -0.04 & 0.06 & 0.03 & -0.02 & -0.03 & 0.04 & 0.03 & -0.02 & -0.03 & 0.04 & 0.03 & -0.02 & -0.03 & 0.04 & 0.03 \\
\hline$\omega L_{a d}$ & -0.08 & -3.51 & 0.01 & 0.01 & 3.25 & 1.84 & 0.00 & 0.00 & 5.17 & 5.17 & 0.00 & 0.01 & 5.20 & 4.76 & 0.00 & 0.00 \\
\hline$R_{f}$ & 0.41 & 0.12 & 0.08 & 0.02 & -0.03 & 0.01 & 0.01 & 0.01 & -0.08 & -0.22 & 0.02 & 0.09 & -0.03 & -0.03 & 0.01 & 0.02 \\
\hline$R_{D}$ & -0.03 & -0.01 & 0.00 & 0.00 & -0.01 & -0.01 & 0.00 & 0.00 & -0.01 & -0.02 & 0.00 & 0.00 & -0.01 & -0.01 & 0.00 & 0.00 \\
\hline$\omega L_{a q}$ & 0.03 & 0.00 & 0.00 & 0.00 & -0.01 & 0.01 & 0.00 & 0.00 & -0.01 & 0.01 & 0.00 & 0.00 & 0.00 & 0.01 & 0.00 & 0.00 \\
\hline$R_{Q}$ & -0.01 & 0.00 & 0.00 & 0.00 & -0.03 & -0.02 & 0.00 & 0.00 & -0.03 & -0.01 & 0.00 & 0.00 & -0.03 & -0.02 & 0.00 & 0.00 \\
\hline$T_{d 0}^{\prime}$ & -0.43 & -1.74 & 0.08 & 0.02 & 1.54 & 0.85 & 0.01 & 0.01 & 2.48 & 2.62 & 0.02 & 0.09 & 2.44 & 2.23 & 0.01 & 0.02 \\
\hline$T_{d}^{\prime}$ & -0.27 & -0.15 & 0.08 & 0.02 & -0.58 & -0.71 & 0.03 & 0.02 & -0.04 & 0.05 & 0.03 & 0.09 & -0.52 & -0.58 & 0.02 & 0.02 \\
\hline$T_{d 0}^{\prime \prime}$ & -0.02 & -1.59 & 0.00 & 0.00 & 1.45 & 0.83 & 0.00 & 0.00 & 2.28 & 2.29 & 0.00 & 0.00 & 2.29 & 2.10 & 0.00 & 0.00 \\
\hline$T_{d}^{\prime \prime}$ & 0.85 & -0.22 & 0.04 & 0.02 & -3.68 & -4.21 & 0.14 & 0.04 & -0.71 & -0.96 & 0.05 & 0.00 & -3.27 & -3.61 & 0.09 & 0.02 \\
\hline$\stackrel{d}{X_{d}^{\prime}}$ & 0.29 & -1.68 & 0.02 & 0.01 & 0.00 & -0.82 & 0.06 & 0.02 & 1.97 & 1.87 & 0.02 & 0.00 & 0.99 & 0.68 & 0.03 & 0.01 \\
\hline$X_{d}^{\prime \prime}$ & 1.15 & -0.31 & 0.06 & 0.04 & -5.06 & -5.78 & 0.20 & 0.06 & -1.01 & -1.36 & 0.07 & 0.01 & -4.50 & -4.95 & 0.12 & 0.03 \\
\hline$T_{q 0}^{\prime \prime}$ & 0.03 & 0.01 & 0.00 & 0.00 & 0.02 & 0.03 & 0.00 & 0.00 & 0.02 & 0.01 & 0.00 & 0.00 & 0.03 & 0.03 & 0.00 & 0.00 \\
\hline $\begin{array}{c}q 0 \\
X_{q}^{\prime \prime} \\
\end{array}$ & 0.53 & -0.07 & 0.02 & 0.02 & -2.35 & -2.65 & 0.09 & 0.03 & -0.56 & -0.71 & 0.03 & 0.00 & -2.13 & -2.32 & 0.05 & 0.01 \\
\hline & & & & & & & High no & e scen & & & & & & & & \\
\hline$\omega L_{d}$ & -0.19 & -0.11 & 0.04 & 0.03 & -1.70 & -0.97 & 0.02 & 0.02 & -4.08 & -4.06 & 0.01 & 0.01 & -3.31 & -3.01 & 0.01 & 0.01 \\
\hline$\omega L_{q}$ & 0.01 & -0.13 & 0.05 & 0.03 & -0.91 & -0.97 & 0.03 & 0.01 & -0.22 & -0.25 & 0.01 & 0.00 & -0.81 & -0.85 & 0.02 & 0.00 \\
\hline$R+3 R_{N}$ & -0.62 & -0.39 & 0.56 & 0.34 & -0.16 & -0.31 & 0.41 & 0.33 & -0.16 & -0.34 & 0.41 & 0.33 & -0.23 & -0.33 & 0.41 & 0.32 \\
\hline$\omega L_{a d}$ & -0.07 & -3.50 & 0.01 & 0.01 & 3.22 & 1.81 & 0.01 & 0.01 & 5.15 & 5.15 & 0.00 & 0.00 & 5.17 & 4.73 & 0.01 & 0.01 \\
\hline$R_{f}$ & 0.24 & 0.32 & 0.06 & 0.06 & -0.11 & 0.11 & 0.03 & 0.03 & -0.27 & 0.03 & 0.08 & 0.11 & -0.09 & 0.06 & 0.03 & 0.03 \\
\hline$R_{D}$ & -0.18 & -0.09 & 0.03 & 0.02 & -0.11 & -0.09 & 0.02 & 0.02 & -0.13 & -0.11 & 0.03 & 0.03 & -0.12 & -0.09 & 0.02 & 0.02 \\
\hline$\omega L_{a q}$ & 0.11 & 0.04 & 0.01 & 0.01 & 0.11 & 0.11 & 0.03 & 0.03 & 0.05 & 0.05 & 0.01 & 0.01 & 0.15 & 0.13 & 0.03 & 0.03 \\
\hline$R_{Q}$ & -0.06 & -0.03 & 0.01 & 0.01 & -0.08 & -0.07 & 0.01 & 0.01 & -0.07 & -0.04 & 0.01 & 0.01 & -0.10 & -0.08 & 0.02 & 0.02 \\
\hline$T_{d 0}^{\prime}$ & -0.26 & -1.93 & 0.06 & 0.05 & 1.61 & 0.74 & 0.04 & 0.03 & 2.67 & 2.37 & 0.08 & 0.11 & 2.49 & 2.14 & 0.03 & 0.03 \\
\hline$T_{d}^{\prime}$ & -0.30 & -0.46 & 0.04 & 0.05 & -0.61 & -0.87 & 0.07 & 0.04 & 0.10 & -0.22 & 0.09 & 0.12 & -0.58 & -0.75 & 0.06 & 0.05 \\
\hline$T_{d 0}^{\prime \prime}$ & 0.14 & -1.51 & 0.03 & 0.02 & 1.54 & 0.89 & 0.02 & 0.02 & 2.40 & 2.37 & 0.03 & 0.03 & 2.38 & 2.17 & 0.02 & 0.02 \\
\hline$T_{d}^{\prime \prime}$ & -0.22 & -0.74 & 0.22 & 0.15 & -4.21 & -4.52 & 0.22 & 0.11 & -0.87 & -1.05 & 0.07 & 0.02 & -3.90 & -4.01 & 0.19 & 0.11 \\
\hline$X_{d}^{\prime}$ & -0.19 & -1.92 & 0.10 & 0.07 & -0.25 & -0.98 & 0.10 & 0.05 & 1.85 & 1.79 & 0.04 & 0.02 & 0.71 & 0.48 & 0.08 & 0.06 \\
\hline$X_{d}^{\prime \prime}$ & -0.55 & -1.16 & 0.35 & 0.24 & -5.89 & -6.30 & 0.33 & 0.17 & -1.40 & -1.61 & 0.14 & 0.07 & -5.47 & -5.59 & 0.28 & 0.18 \\
\hline$T_{q 0}^{\prime \prime}$ & 0.13 & 0.06 & 0.02 & 0.01 & 0.16 & 0.14 & 0.03 & 0.03 & 0.10 & 0.08 & 0.02 & 0.02 & 0.20 & 0.17 & 0.04 & 0.04 \\
\hline$X_{q}^{\prime \prime}$ & -0.22 & -0.44 & 0.15 & 0.11 & -2.70 & -2.86 & 0.14 & 0.07 & -0.72 & -0.81 & 0.06 & 0.03 & -2.54 & -2.59 & 0.12 & 0.08 \\
\hline
\end{tabular}


and the saturation level, as one would expect. This fact is clearly seen in results of case 3, which has the largest errors for $\omega L_{d}, \omega L_{a d}$ but the lowest saturation level, and must be investigated further.

\section{CONCLUSION}

The focus of this paper was the investigation and implementation of a procedure for reliable parameter identification for salient pole synchronous machines that can be used for condition monitoring, online assessment of the power grid, and adaptive control. Focus is given to a procedure that can: 1) run with the machine online, and without taking it out of service, performing difficult and time-consuming tests or involving large perturbations; 2) use common measurements available in a power plant without installing additional transducers in the machine; 3 ) be robust to noise and deviations in measurements caused by uncertainty of instruments or effects not included in the model.

In order to achieve that, a KF was implemented to filter and correct measurements using prior knowledge about the synchronous machine model and its parameters. Validation of the KF shows good correlation with the SM model available in SPS. The results also demonstrates the correlation is sensitive to saturation effects, but insensitive to the load condition. The goodness of fit is maintained under a "standard" noise scenario. As one would expect, performance degrades in the "high" noise scenario, specially when the SNR is extremely low.

Outputs of the KF are then fed into a RLS algorithm that is able to reliably identify 8 out of 13 parameters from the SM: $R+3 R_{N}, R_{f}, R_{D}, R_{Q}, \omega L_{d}, \omega L_{a d}, \omega L_{q}, \omega L_{a q}$. The parameters not being estimated are: $R, L_{0}+3 L_{N}, l_{f}, l_{D}, l_{Q}$, in which $R, L_{0}+3 L_{N}$ are not relevant for calculation of standard parameters and the leakage reactances $l_{f}, l_{D}, l_{Q}$ can be assumed as constants.

Despite current limitations, results are promising and, when validated experimentally, the proposed procedure might be used for practical condition monitoring applications, such as detection of broken damper winding, turn-to-turn short circuit and air-gap eccentricity. Another possibility is using the proposed procedure to calculate standard parameters in multiple load conditions based on measurements from existing protection IEDs, without the use of special test equipment.

\section{REFERENCES}

[1] NVE, "Vannkraftpotensialet," https://www.nve.no/energiforsyning-ogkonsesjon/vannkraft/vannkraftpotensialet/, Dec. 2017.

[2] IEEE, Std 1110-2002 IEEE Guide for Synchronous Generator Modeling Practices and Applications in Power System Stability Analyses. New York: IEEE, Nov. 2003.

[3] I. M. Canay, "Causes of Discrepancies on Calculation of Rotor Quantities and Exact Equivalent Diagrams of the Synchronous Machine," IEEE Trans. Power Appar. Syst., vol. PAS-88, no. 7, pp. 1114-1120, Jul. 1969.

[4] W. Jackson and R. Winchester, "Direct- and Quadrature-Axis Equivalent Circuits for Solid-Rotor Turbine Generators," IEEE Trans. Power Appar. Syst., vol. PAS-88, no. 7, pp. 1121-1136, Jul. 1969.

[5] N. Bianchi, Electrical Machine Analysis Using Finite Elements, ser. Power Electronics and Applications. Boca Raton, FL: Taylor \& Francis, 2005, oCLC: ocm57694820.
[6] J. Lidenholm and U. Lundin, "Estimation of Hydropower Generator Parameters Through Field Simulations of Standard Tests," IEEE Trans. Energy Convers., vol. 25, no. 4, pp. 931-939, Dec. 2010.

[7] IEEE, Std 115-2009 IEEE Guide for Test Procedures for Synchronous Machines. New York: IEEE, May 2010, oCLC: 958760789.

[8] IEC, IEC 60034-4 Rotating Electrical Machines - Part 4: Methods for Determining Synchronous Machine Quantities from Tests, 3rd ed. IEC, May 2008.

[9] F. P. de Mello and L. N. Hannett, "Representation of Saturation in Synchronous Machines," IEEE Trans. Power Syst., vol. 1, no. 4, pp. 8-14, 1986.

[10] J. Pyrhonen, T. Jokinen, and V. Hrabovcová, Design of Rotating Electrical Machines, 2nd ed. Chichester, West Sussex, United Kingdom: Wiley, 2013.

[11] Statnett, "Funksjonskrav i kraftsystemet," 2012.

[12] E. Kyriakides, G. Heydt, and V. Vittal, "Online Parameter Estimation of Round Rotor Synchronous Generators Including Magnetic Saturation," IEEE Trans. Energy Convers., vol. 20, no. 3, pp. 529-537, Sep. 2005.

[13] M. Cisneros-González, C. Hernandez, R. Morales-Caporal, E. BonillaHuerta, and M. A. Arjona, "Parameter Estimation of a SynchronousGenerator Two-Axis Model Based on the Standstill Chirp Test," IEEE Trans. Energy Convers., vol. 28, no. 1, pp. 44-51, Mar. 2013.

[14] B. Zaker, G. B. Gharehpetian, M. Karrari, and N. Moaddabi, "Simultaneous Parameter Identification of Synchronous Generator and Excitation System Using Online Measurements," IEEE Trans. Smart Grid, vol. 7, no. 3, pp. 1230-1238, May 2016.

[15] L. Ljung, "Lennart Ljung on System Identification Toolbox: History and Development - Video," https://www.mathworks.com/videos/lennartljung-on-system-identification-toolbox-history-and-development96989.html, Apr. 2012.

[16] J. Machowski, J. W. Bialek, and J. R. Bumby, Power System Dynamics: Stability and Control, 2nd ed. Chichester, U.K: Wiley, 2008, oCLC: ocn232130756.

[17] A. Barakat, S. Tnani, G. Champenois, and E. Mouni, "Analysis of synchronous machine modeling for simulation and industrial applications," Simul. Model. Pract. Theory, vol. 18, no. 9, pp. 1382-1396, Oct. 2010.

[18] T. Glad and L. Ljung, Control Theory: Multivariable and Nonlinear Methods. London: Taylor \& Francis, 2000, oCLC: 247761966.

[19] Hydro-Quebec, "Simscape Electrical Reference (Specialized Power Systems)," Mar. 2019.

[20] P. Kundur, N. J. Balu, and M. G. Lauby, Power System Stability and Control, ser. The EPRI Power System Engineering. New York: McGraw-Hill, 1994.

[21] IEEE, Std 421.5-2016: IEEE Recommended Practice for Excitation System Models for Power System Stability Studies, 2016th ed., ser. 421. New York: IEEE, Aug. 2016, no. 5.

[22] Y. Cui and R. Kavasseri, "Particle filter-based dual estimation for synchronous generators," IET Gener. Transm. Distrib., vol. 11, no. 7 , pp. 1701-1708, May 2017.

[23] H. Karayaka, A. Keyhani, G. Heydt, B. Agrawal, and D. Selin, "Synchronous generator model identification and parameter estimation from operating data," IEEE Trans. Energy Convers., vol. 18, no. 1, pp. 121126, Mar. 2003.

[24] M. Dehghani and S. Nikravesh, "Nonlinear state space model identification of synchronous generators," Electr. Power Syst. Res., vol. 78, no. 5, pp. 926-940, May 2008.

[25] L. Ljung, System Identification: Theory for the User, 2nd ed., ser. Prentice Hall Information and System Sciences. Upper Saddle River, NJ: Prentice Hall PTR, 1999.

[26] E. Ghahremani and I. Kamwa, "Online State Estimation of a Synchronous Generator Using Unscented Kalman Filter From Phasor Measurements Units," IEEE Trans. Energy Convers., vol. 26, no. 4, pp. 10991108, Dec. 2011

[27] H. Rehaoulia, H. Henao, and G. Capolino, "Modeling of synchronous machines with magnetic saturation," Electr. Power Syst. Res., vol. 77, no. 5-6, pp. 652-659, Apr. 2007.

[28] B. Zaker, G. B. Gharehpetian, and M. Karrari, "Improving Synchronous Generator Parameters Estimation Using d- q Axes Tests and Considering Saturation Effect," IEEE Trans. Ind. Inf., vol. 14, no. 5, pp. 1898-1908, May 2018. 